\title{
CHARACTERISTIC DIRECTIONS OF CLOSED PLANAR HOMOTHETIC MOTIONS
}

\author{
Ayhan $\operatorname{Tutar}^{1} \S$, Önder Şener ${ }^{2}$, Esra İnan ${ }^{3}$ \\ 1,2,3 Ondokuz Mayis University \\ Arts and Science Faculty \\ Mathematics Department \\ Samsun, 55139, TURKEY
}

\begin{abstract}
In this paper, during one-parameter closed planar homothetic motions, the Steiner area formula and the polar moment of inertia were expressed. The Steiner point or Steiner normal concepts were described according to whether rotation number is different zero or equal to zero, respectively. The moving pole point was given with its components and its relation between Steiner point or Steiner normal was specified. The sagittal motion of a telescopic crane was considered as an example. This motion was described by a double hinge consisting of the fixed control panel of telescopic crane and the moving arm of telescopic crane. The results obtained in the first section of this study were applied for this motion.
\end{abstract}

AMS Subject Classification: 53A17, 70B10

Key Words: Steiner formula, polar moment of inertia, planar kinematics, homothetic motions

\section{Introduction}

For a geometrical object rolling on a line and making a complete turn, some properties of the area of a path of a point were given by [6]. The Steiner area formula and the Holditch theorem during one parameter closed planar homothetic motions were expressed by [8]. We calculated the expression of the

Received: December 30, 2017

(C) 2018 Academic Publications

${ }^{\S}$ Correspondence author 
Steiner formula relative to the moving coordinate system under one parameter closed planar motions. If the points of the moving plane which enclose the same area lie on a circle, then the centre of this circle is called the Steiner point $(h=1),[4],[5]$. If these points lie on a line, we use Steiner normal instead of Steiner point. Then we obtained the moving pole point for the closed planar homothetic motions. We dealt with the polar moment of inertia of a path generated by a closed planar homothetic motion. Furthermore, we expressed the relation between the area enclosed by a path and the polar moment of inertia. As an example, the sagittal motion of a telescopic crane which is described by a double hinge being fixed and moving was considered. The Steiner area formula, the moving pole point and the polar moment of inertia were calculated for this motion. Moreover, the relation between the Steiner formula and the polar moment of inertia was expressed.

\section{Closed Paths in Planar Homothetic Motion}

We consider one parameter closed planar homothetic motion between two reference systems: the fixed $E^{\prime}$ and the moving $E$, with their origins $\left(O^{\prime}, O\right)$ and orientations. Then, we take into account motion relative to the fixed coordinate system (direct motion).

By taking displacement vector $O O^{\prime}=U$ and $O^{\prime} O=U^{\prime}$, the total angle of rotation $\alpha(t)$, the motion defined by the transformation

$$
X^{\prime}(t)=h(t) R(t) X+U^{\prime}(t)
$$

is called one-parameter closed planar homothetic motion and denoted by $E / E^{\prime}$, where $h$ is a homothetic scale of the motion $E / E^{\prime}, X$ and $X^{\prime}$ are the position vectors with respect to the moving and fixed rectangular coordinate systems of a point $X \in E$, respectively. The homothetic scale $h$ and the vectors $X^{\prime}$ and $U, U^{\prime}$ are continuously differentiable functions of a real parameter $t$.

In Eq. (1), $X^{\prime}(t)$ is the trajectory with the respect to the fixed system of a point $X$ belonging to the moving system. If we replace $U^{\prime}=-R U$ in Eq. (1), the motion can be written as

$$
X^{\prime}(t)=R(t)(h(t) X-U(t)) .
$$

Then, we can write as follows the coordinates of the equation above,

$$
\begin{gathered}
X^{\prime}(t)=\left(\begin{array}{l}
x_{1}^{\prime}(t) \\
x_{2}^{\prime}(t)
\end{array}\right), U^{\prime}(t)=\left(\begin{array}{l}
u_{1}^{\prime}(t) \\
u_{2}^{\prime}(t)
\end{array}\right), \\
X=\left(\begin{array}{l}
x_{1} \\
x_{2}
\end{array}\right), U(t)=\left(\begin{array}{l}
u_{1}(t) \\
u_{2}(t)
\end{array}\right)
\end{gathered}
$$


and the rotation matrix as:

$$
R(t)=\left(\begin{array}{cc}
\cos (\alpha(t)) & -\sin (\alpha(t)) \\
\sin (\alpha(t)) & \cos (\alpha(t))
\end{array}\right)
$$

The components of Eq. (1) can be given as

$$
\left.\begin{array}{l}
x_{1}^{\prime}(t)=\cos (\alpha(t))\left(h(t) x_{1}-u_{1}\right)-\sin (\alpha(t))\left(h(t) x_{2}-u_{2}\right), \\
x_{2}^{\prime}(t)=\sin (\alpha(t))\left(h(t) x_{1}-u_{1}\right)+\cos (\alpha(t))\left(h(t) x_{2}-u_{2}\right) .
\end{array}\right\}
$$

From Eq. (4), by differentiation with respect to $t$, we have

$$
\left.\begin{array}{rl}
d x_{1}^{\prime} & =-\sin \alpha\left(h x_{1}-u_{1}\right) d \alpha+\cos \alpha\left(d h x_{1}-d u_{1}\right) \\
& -\cos \alpha\left(h x_{2}-u_{2}\right) d \alpha-\sin \alpha\left(d h x_{2}-d u_{2}\right) \\
d x_{2}^{\prime} & =\cos \alpha\left(h x_{1}-u_{1}\right) d \alpha+\sin \alpha\left(d h x_{1}-d u_{1}\right) \\
& -\sin \alpha\left(h x_{2}-u_{2}\right) d \alpha+\cos \alpha\left(d h x_{2}-d u_{2}\right)
\end{array}\right\}
$$

\subsection{The Steiner Formula in Planar Homothetic Motion}

The formula for the area $F$ of a closed planar curve of the point $X^{\prime}$ is given by

$$
F=\frac{1}{2} \oint\left(x_{1}^{\prime} d x_{2}^{\prime}-x_{2}^{\prime} d x_{1}^{\prime}\right)
$$

If Eqs. (4) and (5) are placed in Eq. (6), we have that:

$$
\begin{aligned}
2 F & =\left(x_{1}^{2}+x_{2}^{2}\right) \oint h^{2} d \alpha+x_{1} \oint\left(-2 h u_{1} d \alpha-h d u_{2}+d h u_{2}\right) \\
& +x_{2} \oint\left(-2 h u_{2} d \alpha+h d u_{1}-d h u_{1}\right) \\
& +\oint\left\{\left(u_{1}^{2}+u_{2}^{2}\right) d \alpha+u_{1} d u_{2}-u_{2} d u_{1}\right\} .
\end{aligned}
$$

The following expressions are used in Eq. (7)

$$
\left.\begin{array}{l}
\oint\left(-2 h u_{1} d \alpha-h d u_{2}+d h u_{2}\right)=a^{*} \\
\oint\left(-2 h u_{2} d \alpha+h d u_{1}-d h u_{1}\right)=b^{*} \\
\oint\left\{\left(u_{1}^{2}+u_{2}^{2}\right) d \alpha+u_{1} d u_{2}-u_{2} d u_{1}\right\}=c .
\end{array}\right\}
$$

The scalar term $c$ which is related to the trajectory of the origin of the moving system may be given as by taking $F_{0}:=F\left(x_{1}=0, x_{2}=0\right)$

$$
2 F_{0}=c .
$$

The coefficient $m$ is defined by

$$
m=\oint h^{2} d \alpha=h^{2}\left(t_{0}\right) \oint d \alpha=h^{2}\left(t_{0}\right) 2 \pi \nu
$$


with the rotation number $\nu$ establishes whether the lines with $F=$ const. describing circles or straight lines. If $\nu \neq 0$, then we have circles. If $\nu=0$, the circles reduce to straight lines. If Eqs. (8), (9) and (10) are substituted in Eq. (7), then

$$
2\left(F-F_{0}\right)=\left(x_{1}^{2}+x_{2}^{2}\right) m+a^{*} x_{1}+b^{*} x_{2}
$$

can be obtained.

\subsubsection{A Different Parametrization for the Integral Coefficients}

Eq. (2) by differentiation with respect to $t$ yields

$$
d X^{\prime}=d R(h X-U)+R(d h X-d U)
$$

and if $X=P=\left(\begin{array}{c}p_{1} \\ p_{2}\end{array}\right)$ (the pole point) is taken,

$$
0=d X^{\prime}=d R(h P-U)+R(d h P-d U)
$$

can be written. Then if $U=\left(\begin{array}{l}u_{1} \\ u_{2}\end{array}\right)$ is solved from Eq. (12), we find

$$
\left.\begin{array}{l}
u_{1}=h p_{1}+p_{2} \frac{d h}{d \alpha}-\frac{d u_{2}}{d \alpha} \\
u_{2}=h p_{2}-p_{1} \frac{d h}{d \alpha}+\frac{d u_{1}}{d \alpha}
\end{array}\right\} .
$$

If Eq. (13) is replaced in Eq. (8),

$$
a^{*}=\oint\left(-2 h^{2} p_{1} d \alpha\right)+\oint\left(-2 h d h p_{2}+h d u_{2}+u_{2} d h\right)
$$

and

$$
b^{*}=\oint\left(-2 h^{2} p_{2} d \alpha\right)+\oint\left(2 h d h p_{1}-h d u_{1}-u_{1} d h\right)
$$

can be rewritten. Also Eqs. (14) and (15) can be expressed as separately

$$
\begin{gathered}
a:=\oint\left(-2 h^{2} p_{1} d \alpha\right), b:=\oint\left(-2 h^{2} p_{2} d \alpha\right) \\
\mu_{1}:=\oint\left(-2 h d h p_{2}+h d u_{2}+u_{2} d h\right), \mu_{2}:=\oint\left(-2 h d h p_{1}-h d u_{1}-u_{1} d h\right),
\end{gathered}
$$

where $\mu=\left(\begin{array}{l}\mu_{1} \\ \mu_{2}\end{array}\right)$.

We found the formula for the area, using Eqs. (16) and (17)

$$
2\left(F-F_{o}\right)=\left(x_{1}^{2}+x_{2}^{2}\right) m+a x_{1}+b x_{2}+\mu_{1} x_{1}+\mu_{2} x_{2} .
$$




\subsection{Steiner Point or Steiner Normal in Planar Homothetic Motion}

By taking $m \neq 0$, the Steiner point $S=\left(s_{1}, s_{2}\right)$ for the closed planar homothetic motion can be written as

$$
s_{j}=\frac{\oint h^{2} p_{j} d \alpha}{\oint h^{2} d \alpha}, \quad j=1,2 .
$$

Then

$$
\oint h^{2} p_{1} d \alpha=s_{1} m, \oint h^{2} p_{2} d \alpha=s_{2} m
$$

are found. If Eq. (20) is replaced in Eq. (16) by considering Eq. (18), we obtain the Steiner area formula as

$$
2\left(F-F_{0}\right)=m\left(x_{1}^{2}+x_{2}^{2}-2 s_{1} x_{1}-2 s_{2} x_{2}\right)+\mu_{1} x_{1}+\mu_{2} x_{2} .
$$

By dividing this by $m$ and by completing the squares, one obtains the equation of a circle,

$$
\begin{aligned}
\left(x_{1}-\left(s_{1}-\frac{\mu_{1}}{2 m}\right)\right)^{2} & +\left(x_{2}-\left(s_{2}-\frac{\mu_{2}}{2 m}\right)\right)^{2} \\
& -\left(s_{1}-\frac{\mu_{1}}{2 m}\right)^{2}-\left(s_{2}-\frac{\mu_{2}}{2 m}\right)^{2}=\frac{2\left(F-F_{0}\right)}{m} .
\end{aligned}
$$

All the fixed points of the moving plane which pass around equal orbit areas under the motion $E / E^{\prime}$ lie on the same circle with the center

$$
M=\left(s_{1}-\frac{\mu_{1}}{2 m}, s_{2}-\frac{\mu_{2}}{2 m}\right)
$$

in the moving plane.

In the case of $h(t)=1$, since $\mu_{1}=\mu_{2}=0$, the point $M$ and the Steiner point $S$ coincide, [3]. Also by taking $m=0$, if it is replaced in Eq. (18), then we have

$$
\left(a+\mu_{1}\right) x_{1}+\left(b+\mu_{2}\right) x_{2}-2\left(F-F_{0}\right)=0 .
$$

Eq. (24) is of a straight line. If no complete loop occurs, then $\eta=0$ and the circles are reduced to straight lines, in other words, circles whose centers lie at infinity. The normal to the lines of equal areas in Eq. (24) is given by

$$
n=\left(\begin{array}{c}
a+\mu_{1} \\
b+\mu_{2}
\end{array}\right)
$$

which is called the Steiner normal [2]. 


\subsection{The Moving Pole Point in Planar Homothetic Motion}

Using Eq. (13), if $P=\left(\begin{array}{l}p_{1} \\ p_{2}\end{array}\right)$ is solved, then at the pole point $P$ of the motion, we get

$$
\left.\begin{array}{l}
p_{1}=\frac{d h\left(d u_{1}-u_{2} d \alpha\right)+h d \alpha\left(d u_{2}+u_{1} d \alpha\right)}{(d h)^{2}+h^{2}(d \alpha)^{2}}, \\
p_{2}=\frac{d h\left(d u_{2}+u_{1} d \alpha\right)-h d \alpha\left(d u_{1}-u_{2} d \alpha\right)}{(d h)^{2}+h^{2}(d \alpha)^{2}} .
\end{array}\right\}
$$

For $m \neq 0$, using Eqs. (10) and (19), we arrive at the relation in Eq. (20) between the Steiner point and the pole point.

For $m=0$, using Eqs. (16) and (25), we arrive at the relation between the Steiner normal and the pole point below:

$$
\left(\begin{array}{l}
a \\
b
\end{array}\right)=\left(\begin{array}{l}
-2 \oint h^{2} p_{1} d \alpha \\
-2 \oint h^{2} p_{2} d \alpha
\end{array}\right)=n-m
$$

\subsection{The Polar Moments of Inertia in Planar Homothetic Motion}

The polar moments of inertia ' $T$ ' symbolizes a path for closed homothetic motions. We find a formula by using $T, m, n$ in this section and we arrive at the relation between the polar moments of inertia ' $T$ ' and the formula of area ' $F$ ' (see Eq. (33)).

A relation between the Steiner formula and the polar moment of inertia around the pole for a moment is to be found in the book by Blaschke and Müller [1], Müller [4] demonstrated a relation to the polar moment of inertia around the origin, Tölke [7] inspected the same for closed functions and Kuruoğlu, Düldül and Tutar [3] generalized Müller's results for homothetic motion.

If we use as $\alpha$ parametrization, we need to calculate

$$
T=\oint\left(\left(x_{1}^{\prime}\right)^{2}+\left(x_{2}^{\prime}\right)^{2}\right) d \alpha
$$

along the path of $X$. Then, using Eq. (4)

$$
T=\left(x_{1}^{2}+x_{2}^{2}\right) m+x_{1} \oint-2 h u_{1} d \alpha+x_{2} \oint-2 h u_{2} d \alpha+\oint\left(u_{1}^{2}+u_{2}^{2}\right) d \alpha
$$

is obtained. 
We need calculate to the polar moments of inertia of the origin of the moving system, therefore $T_{0}:=T\left(x_{1}=0, x_{2}=0\right)$ one obtains

$$
T_{0}=\oint\left(u_{1}^{2}+u_{2}^{2}\right) d \alpha .
$$

If Eq. (30) is replaced in Eq. (29), then we can write

$$
T-T_{0}=\left(x_{1}^{2}+x_{2}^{2}\right) m+x_{1} \oint\left(-2 h u_{1} d \alpha\right)+x_{2} \oint\left(-2 h u_{2} d \alpha\right) .
$$

Also if Eq. (13) is replaced in Eq. (31), then

$$
\begin{aligned}
T-T_{0} & =\left(x_{1}^{2}+x_{2}^{2}\right) m+x_{1} \oint\left(-2 h^{2} p_{1} d \alpha-2 h p_{2} d h+2 h d u_{2}\right) \\
& +x_{2} \oint\left(-2 h^{2} p_{2} d \alpha+2 h p_{1} d h-2 h d u_{1}\right)
\end{aligned}
$$

is found and by considering together with Eqs. (18) and (32), we arrive at the relation between the polar moments of inertia and the formula for the area below:

$$
T-T_{0}=2\left(F-F_{0}\right)+x_{1} \oint\left(h d u_{2}-u_{2} d h\right)+x_{2} \oint\left(-h d u_{1}+u_{1} d h\right) .
$$

\section{Application: The Motion of Telescopic Crane}

In the previous sections we emphasized three concepts: geometrical objects as the Steiner point or the Steiner normal, the pole point, and the polar moments of inertia for closed homothetic motions. In this section, we want to visualize the experimentally measured motion with these objects. Accordingly, we consider these characteristic directions for this motion.

We will show that how the kinematical objects which used in the previous sections can be applied. In the study of Dathe and Gezzi [2], they considered human gait in planar motions. As an example, we have chosen the sagittal part of the movement of the telescopic crane during working. We have chose the telescopic crane, because the arm of telescopic crane can extend or retract during one parameter closed planar homothetic motion. The motion of telescopic crane has a double hinge and 'a double hinge' means that it has two systems, a fixed arm and a moving arm of telescopic crane (Fig. 3.1). There is a control panel of telescopic crane at the origin of fixed system. ' $L$ ' arm can extend or retract by $h$ parameter. 


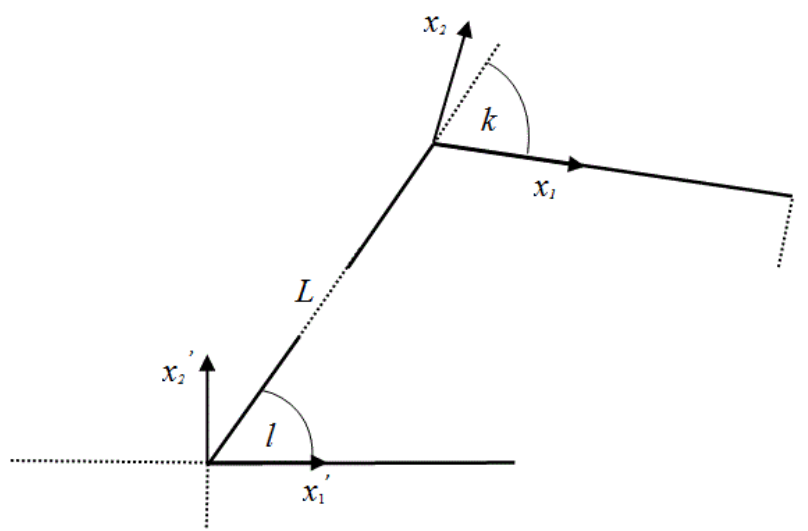

Fig. 3.1: The arms of winch as a double hinge

\subsection{The Mathematical Model}

We start by writing the equations of the double hinge in cartesian coordinates. Then we define using the condition $m=0$, the Steiner normal and the total angle in relation to the double hinge.

By taking

$$
\begin{aligned}
& R(t)=\left(\begin{array}{cc}
\cos (l(t)-k(t)) & -\sin (l(t)-k(t)) \\
\sin (l(t)-k(t)) & \cos (l(t)-k(t))
\end{array}\right), \\
& U^{\prime}(t)=\left(\begin{array}{c}
L \cos (l(t)) \\
L \sin (l(t))
\end{array}\right),
\end{aligned}
$$

we have Eq. (1), namely,

$$
X^{\prime}(t)=h(t) R(t) X+U^{\prime}(t) .
$$

Also we know that $U^{\prime}=-R U$ and then we can write

$$
U(t)=\left(\begin{array}{l}
u_{1}(t) \\
u_{2}(t)
\end{array}\right)=\left(\begin{array}{l}
-L \cos (k(t)) \\
-L \sin (k(t))
\end{array}\right) .
$$

can be written. So the double hinge may be written as

$$
\left.\begin{array}{rl}
x_{1}^{\prime}(t) & =\cos (l(t)-k(t))\left(h(t) x_{1}+L \cos (k)\right) \\
& -\sin (l(t)-k(t))\left(h(t) x_{2}+L \sin (k)\right) \\
x_{2}^{\prime}(t) & =\sin (l(t)-k(t))\left(h(t) x_{1}+L \cos (k)\right) \\
& +\cos (l(t)-k(t))\left(h(t) x_{2}+L \sin (k)\right)
\end{array}\right\},
$$


where $\alpha=l-k$ is the resulting total angle.

We begin by calculating the time derivative of Eq. (36). We obtain the velocities $x_{1}^{\prime}, x_{2}^{\prime}$ which have to be inserted into Eq. (6):

$$
\begin{aligned}
x_{1}^{\prime} x_{2}^{\prime}-x_{2}^{\prime} x_{1}^{\prime} & =\left(h^{2}\left(x_{1}^{2}+x_{2}^{2}\right)+L^{2}\right)(\dot{l}(t)-\dot{k}(t))+L^{2} k(t) \\
& +x_{1}(2 h L \cos (k(t)) \dot{(l}(t)-\dot{k}(t)) \\
& +h L \cos (k(t)) \dot{k(t)}-L d h \sin (k(t)) \\
& +x_{2}(2 h L \sin (k(t)) \dot{(i}(t)-\dot{k}(t)) \\
& +h L \sin (k(t)) \dot{k(t)}+L d h \cos (k(t)) .
\end{aligned}
$$

We now integrate the previous equation using periodic boundary conditions by assuming the integrands as periodic functions. The periodicity of $f$ implies that integrals of the following types vanish

$\oint d f=\oint_{1}^{F} \dot{f} d t=\left.f\right|_{1} ^{F}=0$. As a result of this, some of the integrals of Eq. (37) are not equal to zero and we finally obtain a simplified expression for the area namely

$$
\begin{aligned}
& F=\frac{1}{2} \int_{t_{1}}^{t_{2}}\left(x_{1}^{\prime} x_{2}^{\prime}-x_{2}^{\prime} x_{1}^{\prime}\right) d t \\
2 F= & x_{1} \int_{t_{1}}^{t_{2}} 2 L h(\cos k)(\dot{l}-\dot{k}) d t \\
+ & x_{1} \int_{t_{1}}^{t_{2}} L(h(\cos k) \dot{k}-d h \sin k) d t \\
+ & x_{2} \int_{t_{1}}^{t_{2}} 2 L h(\sin k)(\dot{l}-\dot{k}) d t \\
+ & x_{2} \int_{t_{1}}^{t_{2}} L(h(\sin k) \dot{k}+d h \cos k) d t .
\end{aligned}
$$


We may have the following expressions from Eq. (38)

$$
\left\{\begin{array}{l}
\left.\int_{t_{1}}^{t_{2}} 2 L h(\cos k)(\dot{l}-\dot{k}) d t+\int_{t_{1}}^{t_{2}} L(h(\cos k) \dot{k}-d h \sin k) d t\right)=\bar{a} \\
\left.\int_{t_{1}}^{t_{2}} 2 L h(\sin k)(\dot{l}-\dot{k}) d t+\int_{t_{1}}^{t_{2}} L(h(\sin k) \dot{k}+d h \cos k) d t\right)=\bar{b}
\end{array}\right\} .
$$

Differentiating Eq. (35) with respect to $t$ and then replacing both of them in Eq. (39), Eq. (8) is found for application.

In Section 2.1.1, by using Eq. (13), we find

$$
\left.\begin{array}{l}
\bar{a}=\underbrace{\int_{t_{1}}^{t_{2}}\left(-2 h^{2} p_{1} d \alpha\right)}_{a}+\underbrace{\int_{t_{1}}^{t_{2}}\left(-2 h p_{2} d h+h d u_{2}+u_{2} d h\right)}_{\mu_{1}} \\
\bar{b}=\underbrace{\int_{t_{1}}^{t_{2}}\left(-2 h^{2} p_{2} d \alpha\right)}_{b}+\underbrace{\int_{t_{1}}^{t_{2}}\left(-2 h p_{1} d h+h d u_{1}+u_{1} d h\right)}_{\mu_{2}}
\end{array}\right\}
$$

and we have a straight line below:

$$
2 F=\left(a+\mu_{1}\right) x_{1}+\left(b+\mu_{2}\right) x_{2} .
$$

In this case, we have the Steiner normal

$$
\begin{aligned}
n & =\left(\begin{array}{c}
a+\mu_{1} \\
b+\mu_{2}
\end{array}\right) \\
& =L\left(\begin{array}{c}
\int_{t_{1}}^{t_{2}}\{2 h(\cos k)(\dot{l}-\dot{k})+(h(\cos k) \dot{k}-d h \sin k)\} d t \\
\int_{t_{1}}^{t_{2}}\{2 h(\sin k)(\dot{l}-\dot{k})+(h(\sin k) \dot{k}+d h \cos k)\} d t
\end{array}\right) .
\end{aligned}
$$

\subsection{The Moving Pole Point of the Motion of Telescopic Crane}

If Eq. (35) is replaced in Eq. (26), the pole point $P=\left(\begin{array}{l}p_{1} \\ p_{2}\end{array}\right)$ is found with the components

$$
\left.\begin{array}{l}
p_{1}=\frac{d h(L(\sin k) \dot{l})-h(\dot{l}-\dot{k})(L(\cos k) \dot{l})}{(d h)^{2}+h^{2}(\dot{l}-\dot{k})^{2}} \\
p_{2}=\frac{d h(-L(\cos k) \dot{l})-h(\dot{l}-\dot{k})(L(\sin k) \dot{l})}{(d h)^{2}+h^{2}(\dot{l}-\dot{k})^{2}}
\end{array}\right\}
$$


are found and we can write

$$
P=\left(\begin{array}{c}
p_{1} \\
p_{2}
\end{array}\right)=\frac{\dot{L}}{(d h)^{2}+h^{2}(\dot{l}-\dot{k})^{2}}\left(\begin{array}{c}
d h \sin k-h(\dot{l}-\dot{k}) \cos k \\
-d h \cos k-h(\dot{l}-\dot{k}) \sin k
\end{array}\right) .
$$

Also using Eqs. (40) and (42), we reach the relation between the Steiner normal and the Pole point (Eq. (27)).

\subsection{The Polar Moments of Inertia of the Motion of Telescopic Crane}

Using Eqs. (28) and (36), if Eq. (35) is replaced in Eq. (29), we obtain

$$
T=x_{1} \oint 2 h L(\cos k)(\dot{l}-\dot{k}) d t+x_{2} \oint 2 h L(\sin k)(\dot{l}-\dot{k}) d t .
$$

By considering together with Eqs. (40), (41) and (45), we arrive at the relation between the polar moments of inertia and the formula for the area below:

$$
\begin{aligned}
T & =2 F+x_{1} L \oint(-h(\cos k) \dot{k}+d h \sin k) \\
& -x_{2} L \oint(h(\sin k) \dot{k}+d h \cos k)
\end{aligned}
$$

\section{References}

[1] W. Blaschke and H.R. Müller, Ebene Kinematik, R. Oldenbourg, München (1956).

[2] H. Dathe and R. Gezzi, Characteristic directions of closed planar motions, Zeitschrift für Angewandte Mathematik und Mechanik, 92 (2012), 731-748.

[3] N. Kuruoğlu, M. Düldül and A. Tutar, Generalization of Steiner formula for the homothetic motions on the planar kinematics, Applied Mathematics and Mechanics (Engl. Edition), 24 (2003), 945-949.

[4] H.R. Müller, Verall gemeinerung einer Formel von Steiner, Abh. Braunschweig. Wiss. Ges., 29 (1978), 107-113.

[5] H.R. Müller, Über Trä gheitsmomente bei Steinerscher Massenbelegung, Abh. Braunschweig. Wiss. Ges., 29 (1978), 115-119. 
[6] J. Steiner, Von dem Krümmungs-Schwerpuncte ebener Curven, Journal für die reine und angewandte Mathematik, 21 (1840), 33-63.

[7] J. Tölke, Steiner-Formein für die Bahnflachen geschlossener Aquiaffinbewegungen, Sitzungsber. Ö sterr. Akad. Wiss, Math.-Nat. Klasse, 187 (1978), 325-337.

[8] A. Tutar and N. Kuruoğlu, The Steiner formula and the Holditch theorem for the homothetic motions on the planar kinematics, Mechanism and Machine Theory, 34 (1999), 1-6. 